\title{
The omics era: what can nuclear magnetic resonance tell us on metabolomics?
}

\author{
Franca Castiglione, Monica Ferro, Andrea Mele \\ Department of Chemistry, Materials and Chemical Engineering "Giulio Natta”, Politecnico di Milano, Italy
}

\begin{abstract}
Summary
A brief overview of the potentiality and use of the metabolic fingerprint of a system or biological process is here proposed. The information on the type, quantity and variation of the pool of metabolites and its relationship with a given biological process is commonly referred to as metabolomics. One powerful analytical approach to the detection and quantitation of metabolites is by Nuclear Magnetic Resonance Spectroscopy (NMR). Additionally, the recently introduced High Resolution Magic Angle Spinning (HR-MAS) NMR approach improved dramatically the potentiality of the method allowing direct sampling of ex vivo specimens, such as tissues and cells, without any pre-treatment or extraction steps. The NMR data can be processed towards the target or non-target analysis of the metabolites. The former passes through the identification of all the metabolites, the latter adopts a multivariate statistical approach such as Principal Components Analysis. In this article, the main methodological points of NMR analysis with multivariate statistics are briefly outlined and discussed. A final case-study on the discrimination of healthy and neoplastic tissues via HR-MAS NMR metabolomics is reported as a paradigmatic application.
\end{abstract}

\section{Introduction}

The -omics approaches, rapidly emerging in the last two decades, include genomics, transcriptomics, proteomics, and

\footnotetext{
Correspondence: Andrea Mele, Department of Chemistry, Materials and Chemical Engineering "Giulio Natta", Politecnico di Milano, Piazza L. da Vinci 32, 20132 Milan, Italy.

Tel.: +39.02.23993006.

E-mail: andrea.mele@polimi.it

Key words: metabolomics, metabolites, NMR, HR-MAS, PCA.

Contributions: the authors contributed equally.

Conflict of interest: the authors declare no potential conflict of interest.

Received for publication: 15 February 2018.

Accepted for publication: 15 February 2018.

(C) Copyright F. Castiglione et al., 2017

Licensee PAGEPress, Italy

Microbiologia Medica 2017; 32:7330

doi: $10.4081 / \mathrm{mm} .2017 .7330$

This article is distributed under the terms of the Creative Commons Attribution Noncommercial License (by-nc 4.0) which permits any noncommercial use, distribution, and reproduction in any medium, provided the original author(s) and source are credited.
}

metabolomics, are important tools aimed to understand the complex biological architecture of an organism and its response to environmental stimuli or induced genetic perturbations. All these approaches, assembled as system biology (19) framework, relay on a common strategy based on the combination of sophisticated analytical techniques with the application of statistical multi-variate methods for data analysis. The term metabolome was introduced for the first time by Oliver et al. in 1998 and was used to describe the metabolite pool of living yeast cells (30). Nowadays metabolomics is broadly defined as the systematic identification and quantification of the small molecules, commonly known as metabolites (product of the metabolism) of any biological system such as cell, tissue, organ, biological fluid, or organism at a specific point in time. Consequently, metabolite analysis is like a snapshot, showing which compounds are present and at what relative levels. Metabolites have a vast range of chemical structures (peptides, oligonucleotides, sugars, organic acids, ketones, aldehydes, amines, amino acids, lipids, steroids, alkaloids, and drugs) and properties. Their molecular weights span two orders of magnitude (30-3000 Da).

The connection between the -omics technologies is shown in Figure 1. Metabolomics is located in the end terminal of the chain, completing the metabolic map (17), while genome and proteome catalyse the chemistry of small molecules. Metabolomics, unlike other omics, is a powerful approach because a change in metabolites concentrations is a direct consequence of protein activity changes (not necessarily true for genomic or proteomic changes). Transcriptomes $(11,15)$ or proteomes are not able to monitor the cell function because there is no simple relationship between mRNA or protein levels and metabolism. Moreover disease (2), environmental factors, drugs $(24,41,45)$, etc., perturbs the state of the metabolome, thus providing a system-wide view of the organism or cell's response. Metabolomics focuses on simple cell systems such as microbiology (42) and plant biochemistry. Metabonomics $(9,23,33)$ (another aspect of metabolomics) studies the metabolic response of animal biochemistry to external stimuli or genetic modification. Despite its relative youth, in comparison to genomics and proteomics, metabolomics is now firmly established as a functional methodology to understanding the molecular complexity of living organisms. Currently, metabolomics research is applied in several fields, from environmental science $(6,27)$, pharmacology $(7,13,39)$ to computer science $(35,37)$ and medicine $(25,32)$ as depicted in Figure 2.

A non-exhaustive list of objectives of metabolomics may include: the assessment of the metabolic profile, i.e. the identification of the collection of molecules originated from a given biochemical process, the detection of any change in the metabolite concentration, the search for biomarkers for the diagnosis and the understanding of complex biochemical mechanism. Depending on the details required about the biological process, there are two main methodologies used in metabolomics. One general method is the target analysis: it comprises the quantification and qualitative analysis of all the metabolites present in a biological system. The 
other general method is a non-target analysis, an approach that includes the metabolic profiling where a large number of metabolites are qualitative detected and then classified using chemometric methods. On the other hand, metabolite profiling follows the fate of a more limited set of metabolites through specific pathways (29).

A variety of analytical platform can be used for metabolite detection. A large class of them are based on hyphenated tech- niques, like gas chromatography-mass spectrometry (GC-MS) and high performance liquid chromatography-mass spectrometry (HPLC-MS), or Nuclear Magnetic Resonance (NMR) spectroscopy. This latter is the main focus of the present article. NMR spectroscopy is one of the most used in the metabolomics field $(19,20,28)$. NMR spectroscopy has unique features including excellent reproducibility, quantitative nature and ability to identify

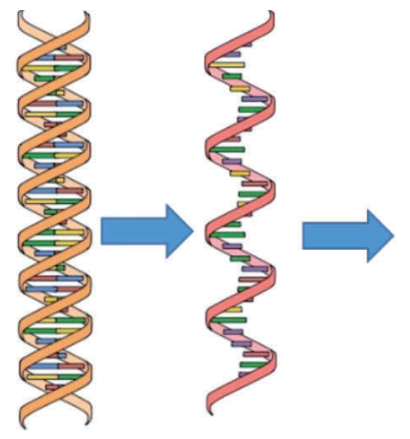

DNA

RNA
A

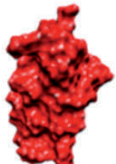

Protein

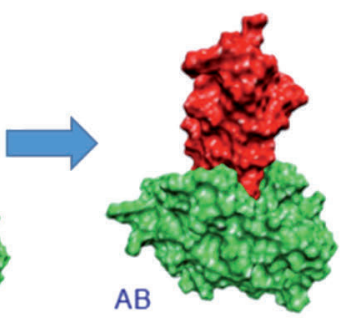

Protein/Protein

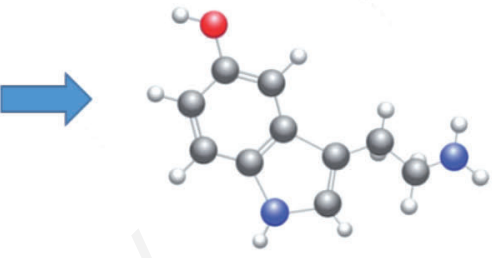

Small Molecule Metabolites

GENOMICS

PROTEOMICS

\section{METABOLOMICS}

\section{Functional Proteomics/Genomics}

Figure 1. Sketch of the relationships among the different types of-omics.

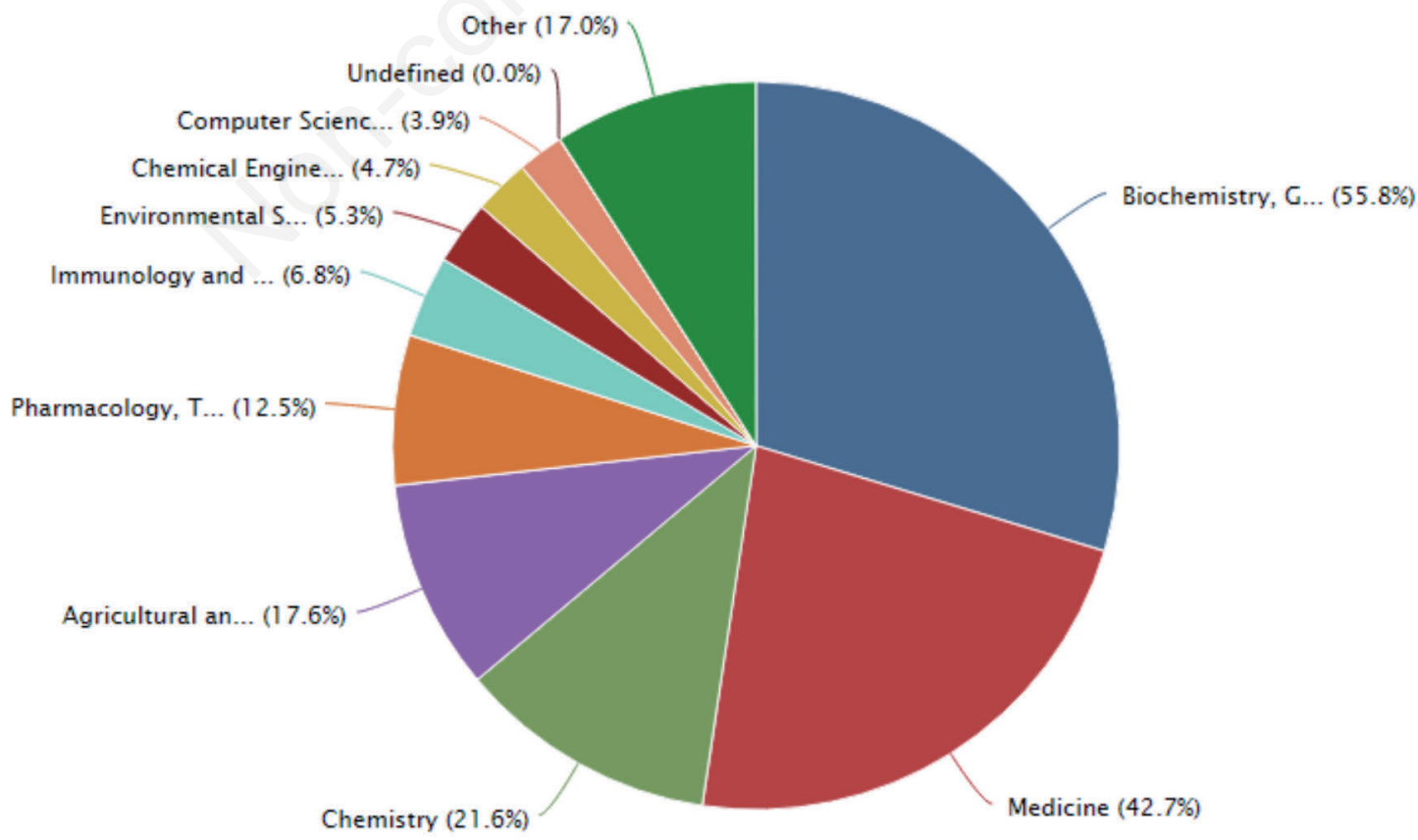

Figure 2. Cake-plot of the impact of metabolomics in the different fields of pure and applied research. The plot is based on the number of peer reviewed publications on metabolomics taken from Scopus database. 
unknown compound through the application of a large variety of correlation experiments able to disentangle complex spectra, thus leading to efficient spectral assignment (19). This review mainly focuses on the use of NMR for metabolomics. A chemical point of view is here proposed in order to disseminate the potentiality that such a spectroscopic approach may have for the targets of medical research and clinic. The paper is structured as follows: i) key concepts of NMR spectroscopy, ii) how to use NMR data for metabolomics; iii) how to process NMR data to obtain information helpful for clinic and diagnosis and iv) illustration of a paradigmatic case study.

\section{Key concepts of nuclear magnetic resonance: imaging $v s$ spectroscopy}

Magnetic resonance is a general physical approach based on the properties of many atomic nuclei $-{ }^{1} \mathrm{H}$ is indeed the most popular - in the presence of a strong external magnetic field, commonly produced via a superconducting magnet. The property exploited is the orientation of the nuclear spin in a magnetic field. The physics behind this phenomenon is complicated and beyond the purpose of this paper, but suffice it to mention here the very basic principle: the two possible orientation of the ${ }^{1} \mathrm{H}$ nuclear spin vectors in a magnetic fields correspond to two different energy levels; the transition between one level and the other can be stimulated by a radiofrequency. Once the system has been perturbed in this way, relaxation phenomena take place and the population of nuclear spins goes back to the initial thermal equilibrium state. The pathway described above can be monitored by modern NMR machines, thus providing a signal that can be elaborated to extract information on the molecular environment that produced the signal itself. According to the type of parameter observed - relaxation of water molecules or the frequency of the energy transitions - and to the type of instrument used - a tomograph or a spectrometer - an image of an organ/tissue or a spectrum can be obtained. The NMR spectrum is basically a plot of the absorption intensity $v s$ the frequency corresponding to such transition. The spectrum is thus made of a baseline where no absorption takes place and some absorption bands, in turn characterized by their position (the frequency of the transition), the intensity (useful for quantitative analysis), the multiplicity (the internal structure of the peak determined by the spin-spin coupling phenomena and strictly dependent on the molecular structure) (40). In the present paper, only NMR spectra will be considered and used as a metabolic fingerprint of the investigated systems. The usual way of obtaining NMR spectra of metabolites generally requires an extraction step, e.g. a cell culture is treated with suitable solvents to extract the metabolic pool, that the solvent is removed and the metabolites are re-dissolved in a second solvent, suitable for NMR data acquisition. In the past decades, a brand new approach has been introduced, allowing the obtainment of high resolution spectra of the metabolites directly from intact cells or tissues (e.g. from histology) without any sample manipulation, namely by skipping the solvent extraction steps. This approach relies upon a technique referred to as High Resolution Magic Angle Spinning (HR-MAS) NMR spectroscopy $(10,22)$. HR-MAS can be seen as a hybrid approach between solution state NMR and solid state NMR. In few words, HR-MAS NMR instrumentation is able to extract the high resolution NMR profile of small molecules (such as the metabolites) out a complex matrix, like suspensions, slurries, polymeric scaffolds or more complex systems like intact cells or even tissues. The introduction of such technology represented a breakthrough in biomedical sci- ences, opening the possibility to observe a chemical fingerprint (the NMR spectrum of a pool of small molecules) directly from biological specimens, with no need of pre-treatment and immediately after surgical excision.

\section{How to use nuclear magnetic resonance data for metabolomics}

The main steps in NMR based metabolomics are sample storage, NMR sample preparation, acquisition and processing of spectra according to two different methodological approaches: target and non-target analysis. The different philosophy of these two routes is sketched in Figure 3.

The Figure shows an array of proton NMR spectra in aqueous solution of metabolites extracted from four cell subtypes derived from the olfactory bulb and subventricular zone of mouse brain (5). Eye inspection reveals that the four NMR spectra, corresponding to four different stem cell types, are very similar (similar types of signal in similar positions and with similar intensities), and all of them characterized by severe spectral overlap. In this case, in order to carry out a targeted metabolomics analysis, the spectral assignment is one of the fundamental steps. The spectral assignment is the correspondence between a group of peaks in the spectrum and a molecule, in our case a metabolite. The spectral assignment is often a difficult task and it requires the use of a set of multidimensional NMR experiments to disentangle the peaks overlap and to derive a univocal spectral assignment. As an example, in the left hand side of Figure 1 the same array of spectra mentioned above is reported along with the labels on some selected peaks, as a result of the preliminary work of spectral assignment. The diagnostic signal of the identified metabolites are indicated. In the case the spectral overlap is not too severe, the peaks integral can be related to the amount of metabolite in the sample and a quantitation is, in principle, achievable. In the remain of this paper we will rather focus on the second approach, corresponding to non-target analysis and making use of multivariate statistical processing of the NMR data. A typical graphical output of Principal Component Analysis (PCA) or related methods such as Partial Least Squares-Discriminant Analysis (PLS-DA) is shown in the right hand side of Figure 3. The key step of this approach is the reduction of the NMR data taken from one or more populations into a smaller dataset of variables, referred to as the Principal Components (PCs). PCs are the eigenvectors obtained from the diagonalization of the covariance matrix and account for the variance of the system. The first two/three PCs can be plotted in a so-called score plot (Figure 3) that provide the maximum information content of the data. Each point in the score plot correspond to a sample, and the clustering of points in the score plot indicate samples sharing similar features. In the case of metabolic studies on patients suffering from a given pathology and on a control healthy set, clustering of points may be easily associated to the state (healthy/non-healthy) of the patients. The example shown in Figure 3 accounts for the fact that OECs cell can be discriminated from the other cell types.

\section{Using nuclear magnetic resonance data for PCA/PLS-DA: nuts and bolts}

In order to understand how to convert NMR data into a score plot useful for clinical purposes or as an aid to diagnosis, some steps should be briefly introduced. 


\section{Processing}

NMR spectra contains thousands of signals due to the complexity of the analysed mixtures. Processing is an essential step before performing statistical analysis, especially in the case of a large data set. The processing step used to make samples comparable include: spectra transformation and apodization, phasing, baseline correction, chemical shift calibration, binning or bucketing and data normalization (21). Spectra alignment can be performed using an internal standard peak or the solvent peak. Binning (also called bucketing) allows reducing the number of variables. Binning consists of dividing spectra into small sectors (bins) of different dimensions (commonly $0.04 \mathrm{ppm}$ ) and summing all signals inside the bin to form a new spectrum with fewer variables (Figures 3 and 4). The normalization step is necessary when are present some differences in acquisition parameters such as different number of scans, different spectrometer, sample concentration. After processing, the data are converted in ASCII format and ready to be processed by a chemometrics software (R, MATLAB).

\section{Chemometrics (statistical analysis)}

Multivariate statistical analysis is often used to simplify the dataset extracting the relevant data, developing classification mod- els and identifying differentiating metabolites (36). Numerous unsupervised and supervised methods exist. PCA $(26,43)$ is the most used unsupervised method, it is considered an exploratory method used to emphasize variation and bring out strong patterns in a dataset. It is used to make data easy to explore and visualize. Another unsupervised method for this purpose is the Hierarchical Cluster Analysis (HCA) $(8,44)$. The most used supervised classification methods are k-nearest-Neighbors (kNN) (1,38), Soft Indipendent Modeling of Class Analogy (SIMCA) (12) and Partial Last Squares Discriminant Analysis (PLS-DA) $(3,14)$. The supervised method use the sample class as a variable for establish discrimination rules. Consequently, the classification is better visible than that obtained by a supervised method, but unsupervised method require a validation protocol. Through chemometrics analysis, it is possible to underline easily differences in biochemical environment and consequently identify the spectral regions responsible of variability and thus key metabolites. Once the separation is obtained, further investigations can be done to identify which spectral region and which molecule are responsible for the separation. Investigation can be performed using different approaches. More detailed NMR experiments, especially twodimensional correlation experiments like TOCSY, HSQC, COSY, HMBC (40), can be used to identify unambiguously some metabo-

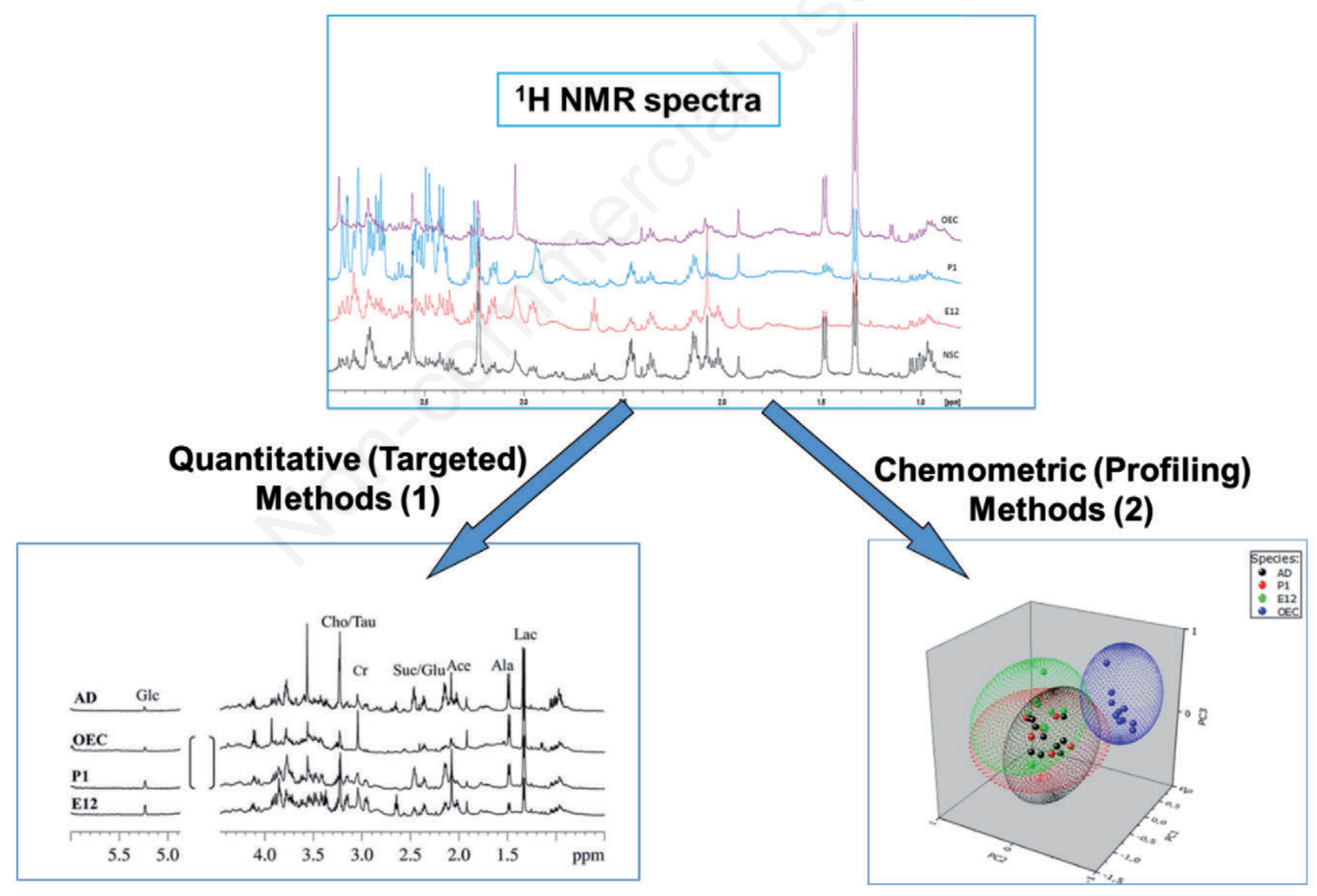

Figure 3. Scheme of different treatment of NMR data according to target (left) and non-target (profiling, right) routes. The top picture shows a stacking of four NMR spectra obtained from the acquous phase after extraction of metabolites of rat brain stem cells (Legend. OECs: Olfactory Ensheathing Cells; P1: postnatal; E12: fetal; NSC: neural stem cells). Bottom left: same spectra after spectral assignment. The main peaks of the identified metabolites (Lactate, Alanine, Acetate, Glutamate, Succinate, Creatinine, Choline, Thaurine, Glucose) are indicated with suitable labels. Bottom right: Three dimensional score plot after PLS-DA analysis of spectral data shown on top. The points in blue are well separated from the remaining points, showing that a discrimination of OECs is possible on the basis of the metabolic profile detected via NMR spectroscopy. Reproduced from Castiglione et al., 2017.5 
lites, additionally databases could provide complementary information (17)

A graphic summary, in the fashion of a flow-chart, of the whole protocol form sample preparation to database search for biomarkers of a given biological process is given in Figure 5 .

\section{HR-MAS nuclear magnetic resonance, metabolomics and multivariate analysis: a case study}

In conclusion, we wish to summarize the concepts introduced above by reporting on a paradigmatic case taken from the literature (34). In this case-study, HR-MAS NMR spectroscopy is used along with chemometrics to discriminate, on the bases of differences of the metabolic fingerprint, healthy and neoplastic human colon tissues. The authors consider the following population and samples: ex vivo samples from 23 subjects, 14 affected by colorectal cancer, 9 healthy. For each person, 5 biopsies were collected, four for the histology and one for NMR analysis. The specimens of the patients with colon cancer were collected from the carcinoma site and $15 \mathrm{~cm}$ away the carcinomas (classified as normal by histological analysis). The NMR spectra obtained on the tissues by HR-MAS NMR showed poor spectra resolution and could not be considered informative as such. The NMR data were then used as input for PCA. However, the score plot of PC1 vs PC2 (namely the two most important principal components) was not able to discriminate healthy and neoplastic categories. Interestingly, the analysis of the subsequent principal components PC3, PC4 and PC5 clearly highlighted two separated clusters of points (Figure 6) corresponding to healthy tissues (upper ellipse) and to neoplastic or histologically normal colon samples.

The subsequent analysis of the loadings provided information on the possible metabolites that can be used as biomarkers for this type of carcinoma. But more interestingly, at least from the metabolomics point of view, is the ability of HR-MAS NMR and PCA di show that the metabolic profile of the tissues taken $15 \mathrm{~cm}$ away from the carcinoma site was statistically more similar to the neoplastic one rather than to the healthy control, thus showing the capability of NMR metabolic analysis to detect pre-malignant metabolic features before any morphological change.

\section{Conclusions and perspectives}

NMR spectroscopy allows to provide precious information for diagnosis and screening based on the metabolic profiles. The joint use of NMR data and multivariate statistics is a powerful predictive tool for a large variety of biological processes. The

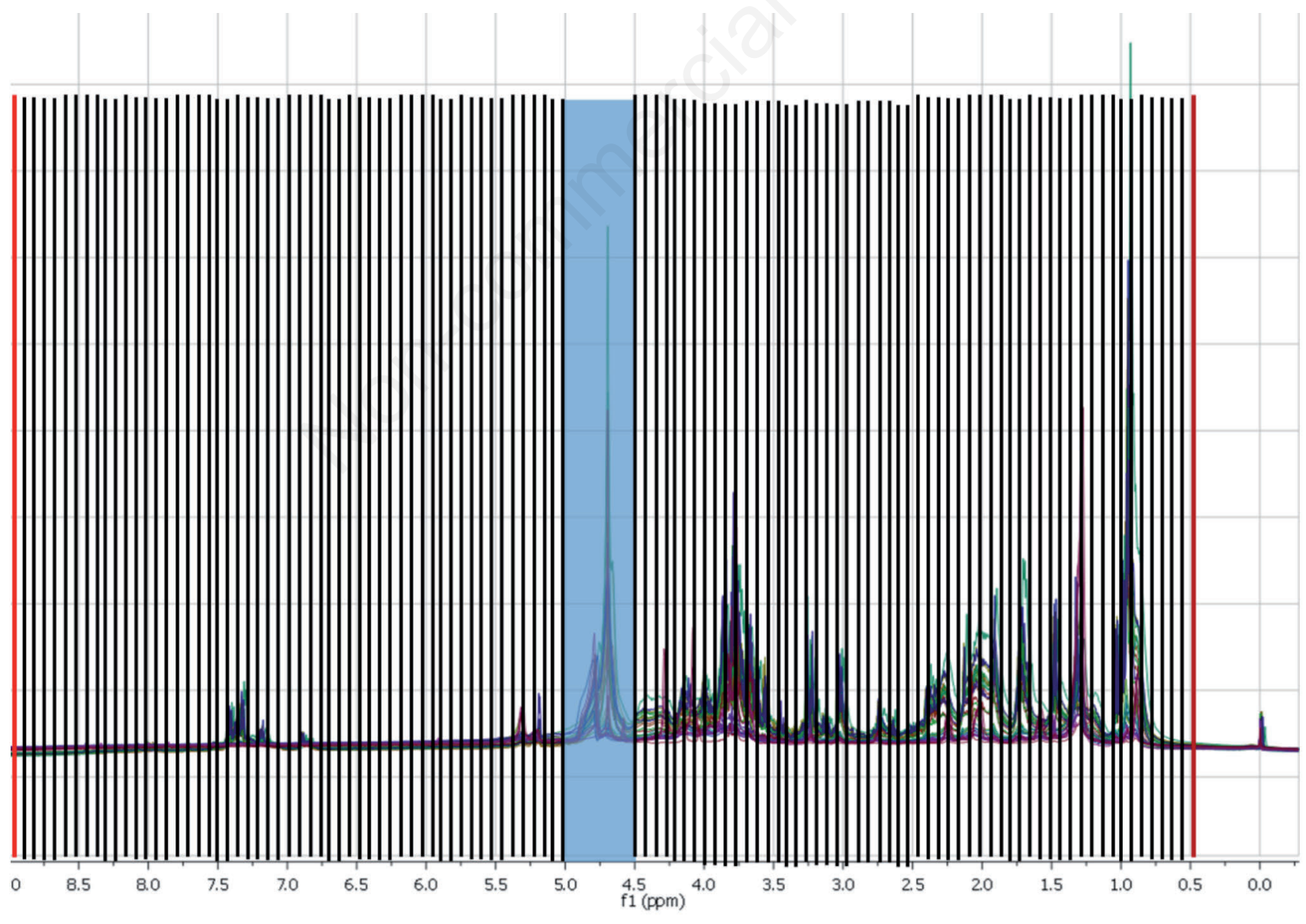

Figure 4. Binning of $0.04 \mathrm{ppm}$ performed on a set of ${ }^{1} \mathrm{H}$ NMR spectra, The region of the solvent (4.5-5 ppm) is highlighted and it is excluded. 


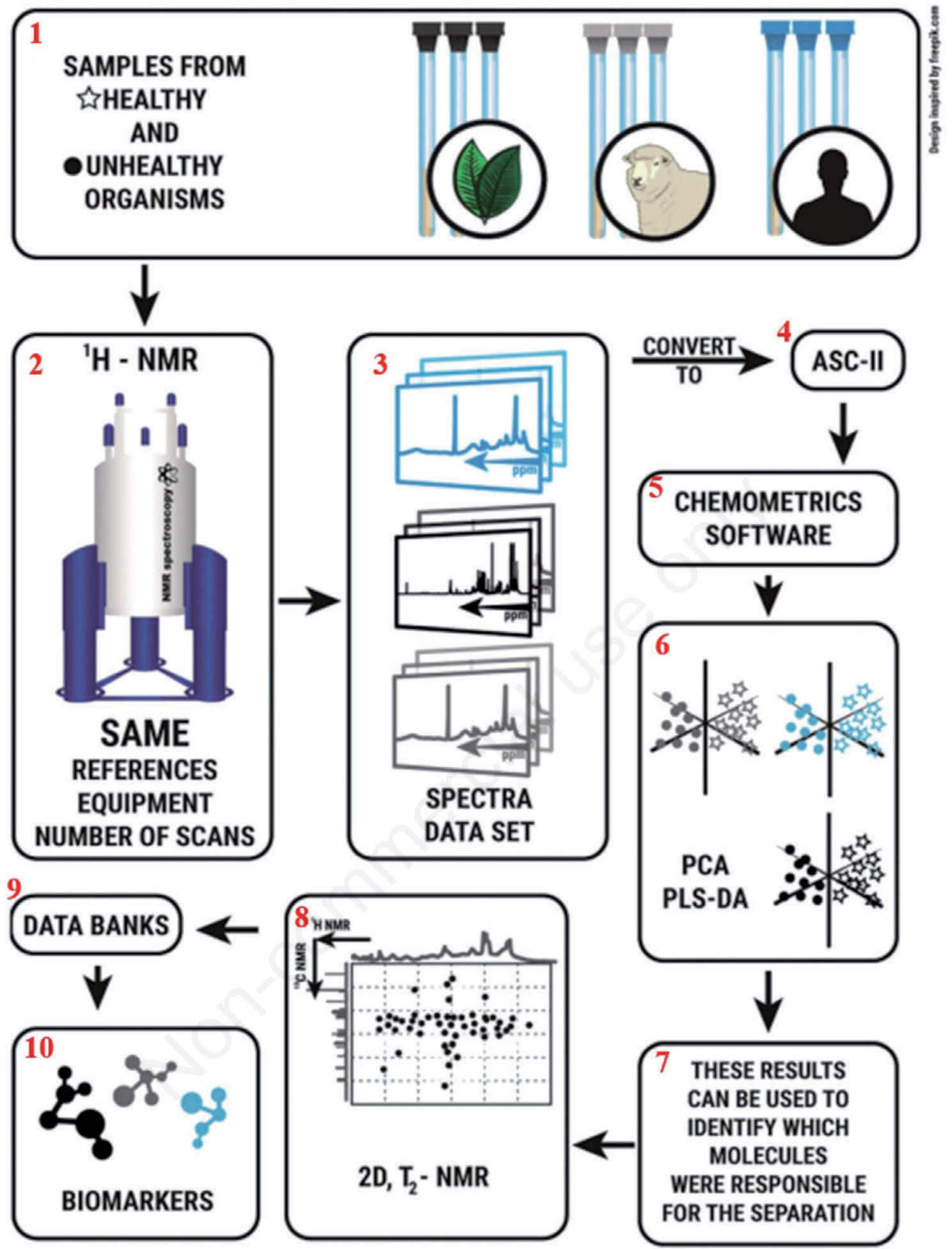

Figure 5. Main steps used in NMR metabolomics. 1) Biological sample preparation. This may pass through extractive procedures or may consists of a mere water solution of the metabolites. In HR-MAS NMR this step is related to the acquisition of ex-vivo specimens or intact cells. 2) The NMD spectra acquisition requires the equipment usually available in the chemistry labs, without any modification of the commercial spectrometers. 3) NMR spectra of a collection of samples are then acquired, with particular care in the choice of the population to screen. 4-6) The NMR data are reduced and undergo PCA or other types of multivariate analysis, affording a twodimensional (or three dimensional) score plot, suitable to observe separation (if any) in clusters. 7-8) The relative contribution of the single bins to the separation of the points in the PC planes are referred to as loadings. Analysis of the loadings may indicate which parts of the NMR spectrum contribute more to the separation of the points in the PC planes. This allows to identify the metabolites that provide the larger contribution to the differentiation of the populations (e.g. healty vs non-healty patients). individuals vs patients. The identification of the metabolites responsible for the classification patterns may be supported by advanced NMR methods, like two-dimensional NMR. 9-10) Finally, a suitable model can be established matching the results of the metabolomics investigation with data base search to identify biomarkers. Reproduced with permission from Pontes et al., 2017.31 Copyright: The Royal Society of Chemistry, 2017. 


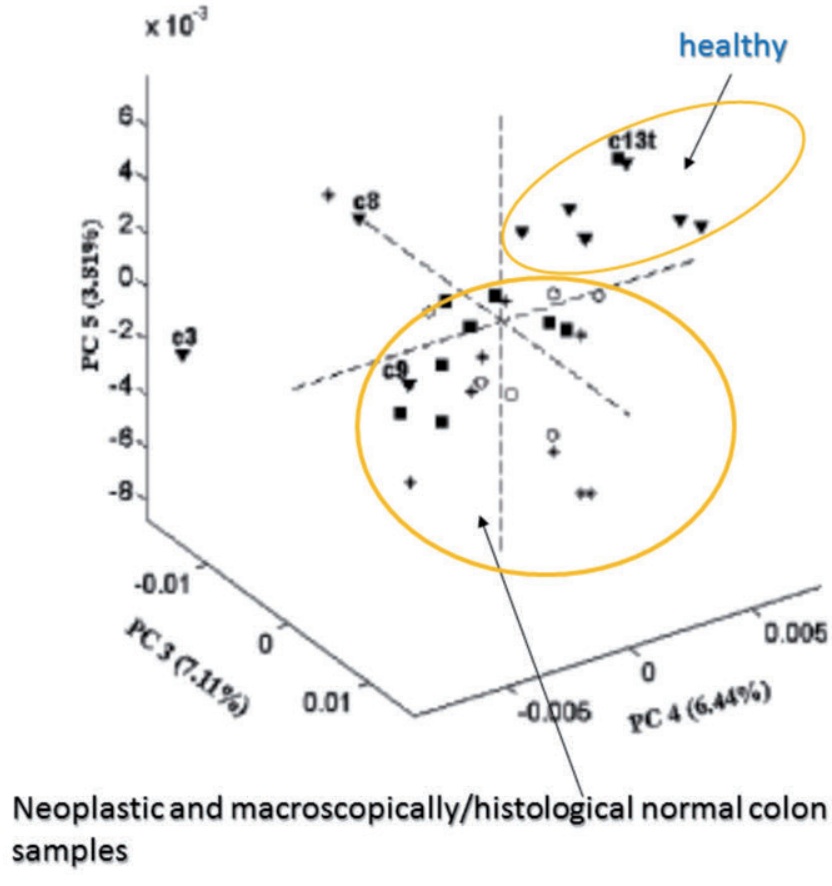

Figure 6. Three-dimensional score plot of PC3 vs PC4 vs PC5 for HR-MAS NMR data of colon tissues. See text for details on sampling. Adapted with permission from Righi et al., 2009.34 Copyright: American Chemical Society, 2009.

metabolomics approach is currently experiencing a dramatic improvement due to the introduction of HR-MAS NMR techniques that make the metabolomics analysis feasible in real time immediately after the surgical excision or biopsy, due to the possibility to use the ex vivo specimen as such, with no intermediate preparation steps. The CARMeN Project (http://www.mnms-platform.com/en/projects/carmen-cancerrmn/) is a clear example of synergy between Chemistry, Spectroscopy and Medical Research towards therapeutic innovation. Within this project, the first clinical HR-MAS NMR spectrometer fully integrated with operating room was installed at the Strasbourg's Hautepierre in 2008.

\section{References}

1. Barba I, De León G, Martín E, et al. Nuclear magnetic resonance-based metabolomics predicts exercise-induced ischemia in patients with suspected coronary artery disease. Magn Reson Med 2008;60:27-32.

2. Benjamin DI Cravatt BF Nomura DK. Global profiling strategies for mapping dysregulated metabolic pathways in cancer. Cell Metabol 2012;16:565-77.

3. Brereton RG, Lloyd GR. Partial least squares discriminant analysis: taking the magic away. J Chemom 2014;28:213-25.

4. Bundy JG Davey MP Viant MR. Environmental metabolomics: a critical review and future perspectives. Metabolomics 2009;5:3-21.

5. Castiglione F, Ferro M, Mavroudakis E, et al. NMR metabolomics for stem cell type discrimination. Sci Rep 2017;7: 155081-12.

6. Castro-Puyana M, Pérez-Míguez R, Montero L, Herrero M.
Application of mass spectrometry-based metabolomics approaches for food safety, quality and traceability. Trends Anal Chem 2017;93:102-18.

7. Christopher LJ, Iyer R, Josephs JL Humphreys WG. Metabolomics-based approaches to determine drug metabolite profiles. RSC Drug Discovery Series 2016;49:247-62.

8. Draisma HHM, Reijmers TH, Meulman JJ, et al. Hierarchical clustering analysis of blood plasma lipidomics profiles from mono-and dizygotic twin families. Eur J Hum Genet 2013;21:95-101.

9. Everett JR. NMR-based pharmacometabonomics: a new paradigm for personalised or precision medicine. Progr Nucl Magn Reson Spectroc 2017;102-103:1-14.

10. Fan TWM, Lane AN. Structure-based profiling of metabolites and isotopomers by NMR. Prog Nucl Magn Reson Spectrosc 2008;52:69-117.

11. Fiehn O. Combining genomics, metabolome analysis, and biochemical modelling to understand metabolic networks. Comp Functi Genom 2001;2:155-68.

12. Geamanu A, Gupta SV, Bauerfeld C, Samavati L. Metabolomics connects aberrant bioenergetic, transmethylation, and gut microbiota in sarcoidosis. Metabolomics 2016;12:1-13.

13. Geenen S, Taylor PN, Snoep JL, et al. Systems biology tools for toxicology. Arch Toxicol 2012;86:1251-71.

14. Gromski PS, Muhamadali H, Ellis DI, et al. A tutorial review: metabolomics and partial least squares-discriminant analysis a marriage of convenience or a shotgun wedding. Anal Chim Acta 2015;879:10-23.

15. Huang S, Yang B, Chen BJ, et al. The emerging role of circular RNAs in transcriptome regulation. Genomics 2017;109:401-7.

16. Johnson CH Ivanisevic J. Siuzdak G. Metabolomics: beyond biomarkers andtowards mechanisms. Nat Rev Mol Cell Biol 2016;17:451-9.

17. Kind T, Scholz M, Fiehn O. How Large Is the Metabolome? A critical analysis of data exchange practices in chemistry. PLoS One 2009;4.

18. Kitano H Systems biology: a brief overview. Science 2002;295:1662-4.

19. Kumar D. Nuclear magnetic resonance (NMR) spectroscopy for metabolic profiling of medicinal plants and their products. Crit Rev Anal Chem 2016;46:400-12.

20. Larive CK, Barding GA, Dinges MM. NMR spectroscopy for metabolomics and metabolic profiling. Anal Chem 2015;87: 133-46.

21. Liland KH. Multivariate methods in metabolomics - from preprocessing to dimension reduction and statistical analysis. Trends Anal Chem 2011;30:827-41.

22. Lindon JC, Beckonert OP, Holmes E, Nicholson JK. High-resolution magic angle spinning NMR spectroscopy: Application to biomedical studies. Prog Nucl Magn Reson Spectrosc 2009;55:79-100.

23. Lindon JC, Nicholson, JK, Holmes E. The handbook of metabolomics and metabonomics. Amsterdam: Elsevier; 2007.

24. Liu W, Deng Y, Liu Y, et al. Stem cell models for drug discovery and toxicology studies. J Biochem Mol Toxicol 2013;27:17-27.

25. Lovestone, S. Searching for biomarkers in neurodegeneration. Nat Med 2010;16:1371-2.

26. Madrid-Gambin F, Garcia-Aloy M, Vázquez-Fresno R, et al. Impact of chlorogenic acids from coffee on urine metabolome in healthy human subjects. Food Res Int 2016;89:1064-70.

27. Mittler R Blumwald E. Genetic engineering for modern agriculture: challenges and perspectives. Ann Rev Plant Biol 2010;61:443-62. 
28. Nagana Gowda GA, Raftery D. Recent advances in NMRbased metabolomics. Anal Chem 2017;89:490-510.

29. Nielsen J, Jewett MC. The role of metabolomics in systems biology. In: Nielsen J, Jewett MC, eds. Metabolomics: a powerful tool in systems biology. Berlin, Heidelberg: Springer; 2007. pp. 1-10.

30. Oliver SG, Winson MK, Kell DB, Baganz F. Systematic functional analysis of the yeast genome. Trends Biotechnol 1998; 16: 373-8.

31. Pontes JGM, Brasil AJM, Cruz GCF, et al. NMR-based metabolomics strategies: plants, animals and humans. Anal Methods 2017;9:1078-96.

32. Preedy VR Patel VB, eds. Biomarkers in disease: methods, discoveries and applications: Biomarkers in cancer. Berlin, Heidelberg: Springer; 2015.

33. Riekeberg E Powers R. New frontiers in metabolomics: from measurement to insight. F1000Research 2017;6:1148.

34. Righi V, Durante C, Cocchi M, et al. Discrimination of healthy and neoplastic human colon tissues by ex vivo HR-MAS NMR spectroscopy and chemometric analyses. J Proteome Res 2009;8:1859-69.

35. Rohart F, Gautier B, Singh A, Lê Cao KA. mixOmics: an R package for omics feature selection and multiple data integration. PLoS Compu Biol 2017;13:e1005752.

36. Saccenti E, Hoefsloot HCJ, Smilde AK, et al. Reflections on univariate and multivariate analysis of metabolomics data. Metabolomics 2014;10:361-74.

37. Salek RM, Haug K, Steinbeck C. Dissemination of metabolomics results: role of MetaboLights and COSMOS. Giga Science 2013;2:8.

38. Sas KM, Karnovsky A, Michailidis G, Pennathur S. Metabolomics and diabetes: analytical and computational approaches. Diabetes 2015;64:718-32.

39. Schnackenberg LK Beger RD. The role of metabolic biomarkers in drug toxicity studies. Toxicol Mechan Methods 2008;18:301-11.

40. Claridge TDW. High-resolution NMR techniques in organic chemistry. 3rd ed. Netherlands: Elsevier Science; 2016.

41. Velculescu VE, Madden SL, Zhang L, et al. Analysis of human transcriptomes. Nat Genet 1999;23:387-8.

42. Villas-Boas SG. Introduction to microbial metabolomics. Microbial Metabolomics: Applications in Clinical, Environmental, and Industrial Microbiology 2016;1-12.

43. Wang $\mathrm{Y}$, Zhao M, Xin Y, et al. 1H NMR and MS based metabolomics study of the therapeutic effect of Cortex Fraxini on hyperuricemic rats. J Ethnopharmacol 2016;185:272-81.

44. Ward JH. Hierarchical grouping to optimize an objective function. J Am Stat Assoc 1963;58:236-44.

45. West PR, Weir AM, Smith AM, et al. Predicting human developmental toxicity of pharmaceuticals using human embryonic stem cells and metabolomics. Toxicol Appl Pharmacol 2010;247:18-27. 